larly around the Mediterranean. The condition is due to an abnormal recessive gene which affects haemoglobin synthesis. Heterozygotes (with only one thalassaemia gene) usually show a mild chronic anaemia with a low haemoglobin concentration in the red cell. This condition, known as thalassaemia minor, can be more serious if the patient also has some abnormal haemoglobins. The homozygous condition known as thalassaemia major or Cooley's anaemia is a very serious disease which is usually fatal before the patient is adult.

The obvious explanation is that this is a serious defect of haemoglobin synthesis in thalassaemics, which have a substantial amount of foetal haemoglobin in their blood. It seemed probable that there was also a defect in the structure of the red cell stroma (the insoluble part of the red cell), for the cells have an abnormally short life-span. Recent work has shown that homozygous thalassaemics cannot synthesize the $\beta$-chain of haemoglobin, and that they also produce much more of the $\alpha$-chain than is necessary for combination with the $\gamma$ - and $\delta$-chains synthesized. Labelling experiments have indicated that, in erythroid cells, the ratio of a-globin to $\gamma$-globin plus $\delta$-globin was $3 \cdot 0$, but this ratio was never found in mature cells. The ratio there was $1 \cdot 0$, corresponding to the presence of normal haemoglobin $\mathrm{A}_{2}$ and haemoglobin $\mathrm{F}$.

It has now been shown (Bergollesi, Pontremoli, Manini and Conconi, European J. Biochem., 3, 364; 1968) that the excess of a-globin formed in reticulocytes from patients with thalassaemia major becomes associated with the stroma. Blood samples from nine patients with thalassaemia major and two normal controls (and bone marrow samples from two patients) were incubated in an amino-acid mixture containing tritium-labelled valine, leucine and lysine. In the control preparations, the activity of both cytoplasmic and stromal proteins increased for two to five hours and then remained constant, with very little label in the stromal proteins. In the patients' samples, the total cell label remained the same after five hours but the label was redistributed. The label in the cytoplasmic proteins increased rapidly for the first five hours and then decreased slowly, while the label in the stromal proteins increased slowly throughout the twenty-five hour incubation period.

Chromatographic separation of the cytoplasmic proteins showed that initially the ratio $a / \gamma+\delta$ globins was $3 \cdot 0$, but as the tritium concentration in the cytoplasm decreased the ratio dropped to $1 \cdot 0$. Nearly all the labelled protein in the stroma was $\alpha$-globin. The authors could find no a-globin in the stroma of mature red cells from thalassaemics, indicating that the a-globin must be further metabolized or firmly associated with the membrane in some way. It is tempting to assume that this reaction is the reason for the fragility of thalassaemic erythrocytes. Certainly further work on this interaction may indicate some of the factors affecting red cell stability.

\section{Regulation of Lambda Genes}

\section{from a Temperate Bacteriophage Correspondent}

It looks as though the temperate coliphage lambda is becoming another gold mine for molecular biologists. The phage has recently been attracting increasing attention, largely because it provides an experimental system for tackling problems of gene regulation at a higher level of complexity than those presented by the venerable lactose operon of $E$. coli. In the lactose operon, a single repressor protein prevents the expres. sion of the three structural genes of the operon; similarly a lambda repressor prevents the expression of the entire phage genome, which has some thirty to fifty genes (see Nature, $21 \%$, $509 ;$ 1968). Unlike the lactose genes, however, the genes of lambda are not all expressed co-ordinately after the repressor is removed. but instead they divide into several groups which differ in both the time and the level of expression during phage growth. Thus current interest in lambda centres on the mechanisms which control this ordered expression. Which lambda genes are controlled directly by the lambda repressor and which are controlled by other means during phage growth ? A possible alternative to direct repression could involve regions of the phage genome which can be transcribed only by a phage specific RN $\Lambda$ polymerase which might appear after the initial stages of infection.

Pereira da Silva and Jacob (Virology, 33, 618; 1967) have just reported an investigation of the control of the expression of two genes, $C_{\mathrm{II}}$ and $O$, which function early in the growth cycle. $C_{\mathrm{II}}$ is involved in the establishment of the lysogenic (totally repressed) state and $O$ is required for the vegetative replication of the phage DNA. Pereira da Silva and Jacob asked whether $C_{\mathrm{II}}$ and $O$ are under direct repressor control or whether they are controlled by some other gene which is in turn under renressor control. Thomas (J. Mol. Biol., 22, 79 ; 1966) has proposed that the $N$ gene, which maps adjacent to the lambda repressor gene and is itself controlled by the repressor, may control the expression of other lambda genes; he suggested that the expression of $O$ requires the presence of the $N$ gene product so that $O$ is only controlled indirectly by the repressor through the mediation of the $N$ protein. Pereira da Silva and Jacob's experiments, however, indicate that both $O$ and $C_{\mathrm{II}}$ are directly controlled by the repressor and that they can be expressed in the absence of the $N$ protein.

In their first experiment, they used lysogenic $E$. coli in which the prophage (the totally repressed phage chromosome) had a defective $N$ gene; they irradiated these cells with ultraviolet light to inactivate the repressor and then showed that the $C_{\mathrm{II}}$ and $O$ functions appeared in the cells. They detected the presence of $C_{\mathrm{II}}$ and $O$ gene products by showing that they were supplied to superinfecting phages which lacked functional $C_{\text {II }}$ and $O$ genes. For both $C_{\text {II }}$ and $O$ the magnitude of the "rescue" effect increased if they delayed superinfec. tion after irradiation, which implies that the $C_{\mathrm{II}}$ and $O$ gene products accumulate after inactivation of the repressor.

In a second and similar experiment they used a cell lysogenic for the defective prophage $t 11$ which, after induction, fails to produce a functional $O$ gene product These rescue experiments showed that the $t 11$ genome also does not produce the $C_{I I}$ function; this finding lends further support to previous suggestions that $C_{\mathrm{I}}$ and $O$ belong to a single operon.

Pereira da Silva and Jacob have thus shown that the $C_{\mathrm{II}}$ and $O$ genes can be expressed in the absence of the $N$ gene product, although their experiments do not eliminate the possibility that the $N$ gene product enhances the expression of $C_{I I}$ and $O$ during normal infection. 